\title{
Antropología, geografía histórica y formación del Estado en México ${ }^{1}$
}

\author{
Pere Sunyer Martín²
}

\begin{abstract}
RESUMEN
En la geografía histórica mexicana se ha dado poco relieve a la aportación que se ha hecho desde la antropología social. Nombres como Miguel Othón de Mendizábal, Paul Kirchhoff, Pedro Armillas, Ángel Palerm y Eric Wolf son los más representativos de esta tradición. En particular el concepto de Mesoamérica propuesto por Kirchhoff en 1943 dio inicio a toda una serie de investigaciones que no solo trataron de llenar de sentido tal término sino que hablaban de la existencia de Estados prehispánicos. Su formación está íntimamente vinculada con la presencia de obras de regadío que permitieron incrementar la producción agrícola y abastecer a una creciente población. Pensar acerca del proceso de conformación del México moderno desde las investigaciones antropológicas sobre el pasado prehispánico, ofrece otro punto de vista del proceso de conformación de los Estados nacionales actuales y su relación con las grandes obras de infraestructuras, como por ejemplo los sistemas de irrigación que fueron construidos con el México nacido de la revolución.
\end{abstract}

Palabras clave: Mesoamérica, irrigación, construcción del Estado moderno.

\begin{abstract}
The Mexican Historical geography hasn't paid enough attention to the contributions made from social anthropology. People such as Miguel Othón de Mendizábal, Paul Kirchhoff, Pedro Armillas, Ángel Palerm and Eric Wolf are among the most outstanding contributors of such an intellectual tradition of social anthropology. In particular, Kirchhoff's concept of Meso-America, coined in 1943, has promoted a whole set of research, which has focused not only on providing meaning to that term, but also on paying more attention to the existence of pre-hispanics States. Its formation is linked to irrigation systems that made it possible to increased crop production and to supply a growing population. To think about the making of modern Mexico from an anthropological research on the prehispanic past provides another point of view on the process of the formation of the current national States and their relationship with large-scale infrastructure irrigation systems that were developed in Mexico after Revolution.
\end{abstract}

Key words: Meso-America, Irrigation, the making of modern State.

1 Artículo recibido el 12 de marzo de 2012, aceptado el 1 de agosto de 2012 y corregido el 1 de octubre de 2012.
2 Universidad Autónoma Metropolitana-Iztapalapa (México). Email: peresunyer@live.com 
La geografía y en particular la geografía histórica tiene en México una larga tradición que ha sido reconocida por diversos investigadores tanto nacionales como extranjeros, si bien el propio término, al menos en el sentido que hoy se le reconoce, no ha tenido la implantación que merecía sino hasta tiempos relativamente recientes ${ }^{3}$. Pese a su nombre, tampoco son los geógrafos quienes han realizado las principales aportaciones. La geografía adolecía, hasta hace relativamente pocos años, de escaso peso específico en el panorama de las ciencias sociales como disciplina que pudiera ir más allá de la actividad docente a la que parecía haber sido consignada. Tampoco han sido siempre los investigadores nacionales quienes se han dado a la tarea de abordar este tema al menos durante una parte relevante del siglo pasado. El descubrimiento de México por parte de Carl Sauer fue el primer paso para que sus discípulos y allegados a la escuela californiana de Berkeley hiciesen de México un lugar de estudio habitual, y lo mismo puede decirse de investigadores procedentes de la escuela geográfica francesa y de otros lares ${ }^{4}$.

Entre los investigadores nacionales, y para el período que va desde mediados del siglo XX hasta aproximadamente los años de 1980, historiadores, arqueólogos y antropólogos sociales son quienes se dedicaron a él con cierto ahínco, y tras ellos, los geógrafos, algunos con formación de historiadores, quienes empezaron a hacer aportaciones al campo, aunque, como se ha dicho, no todos ellos entendieron su contribución como geografía histórica. Ha sido más bien una lectura desde el presente la que permite incluirlas dentro de ella. Es en la actualidad cuando más apor-

3 Con respecto a la larga tradición de esta especialidad en México, hay varios trabajos que han hecho un recuento de su evolución. A los trabajos de Robinson (1972) y García Martínez (1998), hay que añadir los realizados por Moncada (2004), Mendoza y Busto (2010) y Sunyer (2011). En referencia al uso del término "geografía histórica" puede verse Sunyer, (2011, nota 7).

4 Se suele atribuir a la desastrosa $2^{\text {a }}$ Guerra Mundial la llegada masiva de extranjeros a México, quienes fueron acogidos por los gobiernos de Cárdenas, primer, y de Ávila Camacho después. El artículo de García Martínez (1998) y en menor grado el de Sunyer (2011) recogen un número elevado de las aportaciones realizadas en este campo por especialistas extranjeros desde los años de 1950 en adelante. taciones ha recibido la geografía histórica por parte de los geógrafos y cuando más especialistas de disciplinas diferentes participan en su construcción ${ }^{5}$.

En una revisión a lo realizado en México sobre el tema que nos ocupa, el historiador del Colegio de México Bernardo García Martínez en un escrito para la revista Relaciones (Colegio de Michoacán) de 1998, reconocía, un tanto con desolación, que lo realizado hasta el momento, por extranjeros y nacionales, era poco para el conocimiento del país. Al respecto escribía: "Las obras de nuestros días invitan a un balance y permiten ver lo mucho y lo poco que se ha hecho en materia de geografía histórica" (García Martínez, 1998: 55), y abogaba, entre otras demandas, "por una sistematización de los estudios de contenido geográfico". En realidad, a lo que hacía referencia este historiador era que en México faltaban trabajos que permitieran la comprensión integral del territorio, lo que llamaba el "espacio geográfico" mexicano, de la misma manera como habían hecho en los Estados Unidos Ralph H. Brown en Historical Geography of the United States (1949), o Donald W. Meinig en una obra titulada The Shaping of America. A Geographical Perspective of 500 Years History (1986-2002). Finalmente, fue el propio García Martínez quien decidió dedicarse a cubrir las deficiencias que en este tipo de temática existía en el país y parece haber asentado unas ciertas bases para la comprensión de México desde el punto de vista de la geografía histórica ${ }^{6}$.

\footnotetext{
5 En relación a la aportación de los geógrafos. J. Omar Moncada publicó un texto en donde recogía la labor en este campo de los investigadores del Instituto de Geografía de la Universidad Nacional Autónoma de México (Moncada, 2004). Muestra de esta variedad de formaciones fue el "Coloquio Internacional Geocrítica" 2006 dedicado a la "Geografía histórica e historia del territorio" y celebrado en la ciudad de México. Puede verse al respecto el número especial de Scripta Nova dedicado al evento, o bien los comentarios que hice al respecto recientemente (Sunyer, 2011).

6 Dos ejemplos de la influencia de la obra de Bernardo García se puede observar en los mapas de geografía histórica publicados en el Nuevo Atlas $\mathrm{Na}$ cional de México (2007), elaborados por el geógrafo Héctor Mendoza y, más recientemente, el capítulo dedicado a la Geografía histórica de México, elaborado por Mendoza y Busto (2010) publicado en la obra "Construyendo la geografía humana" (Hiernaux, 2010).
} 
Tales reflexiones hay que tenerlas en cuenta, pero da la impresión de que opacan, por un lado, todas las posibilidades que ofrece la geografía histórica en la actualidad, dado el contexto de apertura epistemológica que se está viviendo en la especialidad, más allá de las geografías históricas nacionales; pero, por otro lado, y dentro de la preocupación por lo "nacional", parece desconocerse el esfuerzo hecho por algunos investigadores por comprender la configuración de ese territorio histórico que hoy llamamos México ${ }^{8}$.

En este artículo quiero referirme a una línea de trabajo que merece ocupar, a mi parecer, un lugar más destacado dentro de los estudios geográfico-históricos de este país y que puede ayudar a comprender las características básicas tanto del México prehispánico como las del actual. Concretamente, se trata de las investigaciones que amparadas por la fuerza del concepto de Mesoamérica, propuesto por Paul Kirchhoff en 1943, trataron de aportar elementos que permitieran Ilenarlo de sentido. Las ideas de Vere Gordon Childe, Karl Wittfogel y Julian Steward sirvieron de marco teórico necesario que animó a reconstruir los sistemas de riego prehispánicos, y a que se empezara a hablar de Estados prehispánicos.

Sin embargo, este enfoque teórico tiene mayor interés si lo trasladamos a la comprensión del proceso de conformación de México como Estado moderno, sobre todo al momento en que hubo una apuesta del Estado por impulsar la irrigación del país, desde finales del siglo XIX. Quizás la frase del etnohistoriador Mc C. Adams aparecida en su obra The evolution of Urban Societies: Early Me-

\footnotetext{
7 Reflejo de esa apertura disciplinaria puede verse en los editoriales de algunos de los números del Journal of Historical Geography, como por ejemplo la del primer número escrita por John Patten y Andrew Clark en 1975, el correspondiente al del año de 1987 que Alan R. H. Baker comenzó a dirigir la publicación, y la del año 1997, en la que Michael Heffernan, asume la gestión. En ellas los autores advierten de los cambios que se avecinan para la propia disciplina. Sobre las tendencias de la geografía histórica en el ámbito internacional (léase, Inglaterra, Estados Unidos y Francia) puede verse Sunyer, 2010.

8 Y a cuyo conocimiento, el propio García ha hecho sin duda valiosas aportaciones. Por ejemplo, García (2004, 2008).
}

sopotamia and Pre-Hispanic (Steward, 1966, p. 729) puede servir para entender, en cierta manera, la idea alrededor de la cual va a girar este texto. Dice así: "las instituciones del Estado son la causa de todos los otros cambios, aún del propio Estado" dejando claro la relevancia que pudo haber tenido, de cara al desarrollo cultural, la aparición de una institución como es el Estado. El incremento poblacional, los proyectos de irrigación en gran escala y los logros culturales, son parte de sus consecuencias.

En una primera parte, dedicaré mi atención a conceptos marco que vale la pena explicar, para entender la aportación de los antropólogos a la geografía histórica; presentaré, a continuación, sus principales contribuciones y, para acabar, mostraré algunas ideas que pueden servir para la comprensión de los grandes proyectos nacionales de finales del siglo XIX y principios del XX en México.

\section{Sobre la relación hombre- medio desde la geografía y la antropología}

La relación entre el hombre y el medio natural es uno de los temas centrales de la geografía, que ha tenido su traducción en otras dualidades como hombre-naturaleza, sociedad-territorio, sociedad-medio ambiente. A pesar de la temprana introducción del concepto de "medio" en la geografía por Alejandro de Humboldt para referirse al entorno físico en el que viven los seres vivos (Luis y Urteaga, 1982: s/p; Berdoulay, 1983: s/p), ha sido la geografía humana la que con más interés ha tratado de entenderlo. Conocida es la idea de Carl Ritter con respecto a que la mejor forma de conocer a los pueblos es estudiándolos en "su medio", entendiendo con él, tanto al medio natural, como el social y las estructuras políticas que organiza, aunque concedía un valor especial a la naturaleza en sí (Ritter, 1838: 3-4).

También el proceso histórico que ha seguido tal relación preocupó a los geógrafos del siglo XIX. Friederick Ratzel en su Anthropogeographie, al estudiarla desde una perspectiva que podríamos calificar de ecológica, hablaba de "pueblos de la naturaleza" (Naturvölker) y "pueblos con cultura" (Kulturvölker), una clasificación muy básica que 
aludía a la relación que mantenía el hombre con su entorno, a su desarrollo cultural y a las formas de organización social creadas. En esta tesitura, podríamos incluir la aportación de Paul Vidal de la Blache: el estudio del pays y de los modos de vida (genres de vie) era un recorrido razonado del proceso de adaptación, cultural, de la sociedad al medio que la rodeaba a partir de la ética del libre albedrío $y$ de las posibilidades que ofrece el medio al ser humano.

No es, sin embargo, tal relación un tema exclusivamente geográfico. Como ya ha mostrado Horacio Capel (1987: 23-33), pocas disciplinas han estado tan próximas a la geografía como la antropología, con la que ha compartido preguntas de investigación. En este sentido, no es de extrañar que, tanto desde los estudios físicos del hombre como desde la etnología, haya hecho aportaciones respecto de la relación entre el hombre y el medio, por ejemplo, con Franz Boas, Alfred Kroeber y Julian Steward. Resolver preguntas como ¿en qué momento el hombre dejó de ser "natural" para ser "humano" en el que el denominado "libre albedrío", su "voluntad de ser", que le ha permitido intervenir en su dirección evolutiva, era ya manifiesto? ¿Hasta qué punto este ha sido moldeado por la naturaleza, el medio? ¿En dónde radicaba la libertad humana frente a las constricciones exteriores? Asimismo, desentrañar la esencia de lo cultural y su papel en el proceso de hominización, así como el papel de la difusión cultural en el desarrollo de los pueblos de la Tierra, eran todas ellas significativas para entender al ser humano -en última instancia, lo más importante al decir de Marc Blochpero también su evolución como individuo y sociedad. Y en su solución se encontraron disciplinas diversas, entre ellas la geografía histórica y la antropología, no en vano, la relación hombre-medio y su evolución es de aquellos temas que se encuentran en el límite disciplinario y permiten diferentes formas de abordarse $^{9}$.

La necesidad de encontrar las claves que permitieran comprender la relación histórica

\footnotetext{
9 Y también los historiadores de las ideas, como Clarence Glacken en su obra Traces on the Rhodian Shore (1969), y la historia ambiental se han ocupado de ella (Rucinque y Velásquez, 2007).
}

del hombre con la naturaleza, de la sociedad con su medio ambiente, llevó a la geografía histórica a interesarse por las aportaciones que desde los estudios arqueológicos, etnológicos y antropológicos se hicieron, así como a recurrir a aquellas teorías vigentes que le permitieran entenderla.

La geografía histórica había mostrado una cierta evolución desde el siglo XIX hasta los primeros decenios del XX en los temas abordados y en la forma de hacerlo. Desde aquella subdisciplina preocupada por mostrar la historia del país, el territorio nacional, a partir de la evolución de sus límites, sin más recurso explicativo que el de la propia necesidad humana, del individuo y de la sociedad para desarrollarse ${ }^{10}$, se dio cabida a otro tipo de estudios en los que se justificaba la grandeza nacional a partir de las características físicas y naturales de su territorio y sus efectos sobre los grupos humanos que en él habitaban. El evolucionismo de Darwin en boga y, sobre todo, el darwinismo spencerista, fueron las explicaciones aceptadas entonces. La historia de un país, como parecían decir Halford McKinder o la historiadora Ellen C. Semple, era la del proceso exitoso de adaptación ecológica de sus habitantes a las peculiaridades del entorno; lo mismo sus patrones culturales.

En esta tesitura, los estudios antropológicos podían aportar datos fundamentales para comprender con mayor profundidad la relación hombre-medio, un tema que, desde la antropología social estadounidense, va a ser recurrente como muestran los trabajos de Franz Boas y sus seguidores ${ }^{11}$.

\footnotetext{
${ }^{10}$ Así lo manifestaba Ritter "C'est un des caractères de la nature humaine que, dans chaque homme, est déposée une aptitude spéciale au développement de la quelle il doit sa valeur; et il en est de même de chaque peuple. Le grandeur morale de l'homme consiste dans le développement complet de cette propriété, comme aussi la grandeur nationale et l'originalité des peuples" (Ritter, 1838, p. 3-4) ["Es una de las características de la naturaleza humana que, en cada hombre, se haye depositada una aptitud especial al desarrollo a la cual él debe su valor ; y lo mismo acaece en cada pueblo. La grandeza moral del hombre consiste en el desarrollo completo de esta propiedad, como también la grandeza nacional y la originalidad de los pueblos"].

11 Es conocido el cambio en la posición teórica de Franz Boas -como geógrafo y antropólogo- con respecto a la influencia del medio sobre la actua-
} 
Desde la antropología mexicana, en los años veinte del pasado siglo, ya se había mostrado cierto interés por los aspectos geográficos que afectaban el estudio de las poblaciones, prehispánicas, coloniales y contemporáneas. Por ejemplo, la distribución espacial de aquellos temas que la concernían, como las poblaciones, las etnias, las lenguas. La historia, la etnología y la arqueología de aquellos tiempos conformaban parte de las especialidades que fueron introducidas en el antiguo y porfiriano Museo Nacional de Historia Natural y que conformaron posteriormente el Museo Nacional de Arqueología, Etnología e Historia, institución que acogió a los primeros antropólogos mexicanos como fue el reconocido Manuel Gamio. En este contexto, la obra del etnólogo e historiador Miguel Othón de Mendizábal es emblemática en su preocupación por lo geográfico, y sus escritos están salpicados de todos aquellos aspectos de orden espacial que afectaban las poblaciones prehispánicas o posteriores ${ }^{12}$. De esos estudios quiero destacar en particular el libro titulado "Influencia de la sal en la distribución geográfica de los grupos indígenas de México" (1928) sobradamente citado en los repertorios de geografía histórica en México, aunque menos analizado, que ha sido calificado desde la antropología como una aportación de gran originalidad ${ }^{13}$.

Muchos años más tarde, siguiendo en el marco mexicano, la antropología social volverá a la consideración del medio geográfico a partir de dos conceptos, el de ecología

ción humana, a nivel individual y social, y sus consecuencias en su pensamiento acerca de la forma como evolucionaron los diversos pueblos y culturas del mundo, con su apertura a lo que llamará "relativismo cultural" (Trindell, 1969).

12 Sobre este antropólogo e historiador, puede verse Medina (1998: 159-173).

13 Habría que considerar también la labor efectuada por Robert Redfield (1897-1958) en México, en los años veinte y hasta mediados de los años cuarenta. Este antropólogo norteamericano estuvo fuertemente influido por la escuela sociológica de Chicago, en particular por Robert E. Park, quien era a su vez su suegro, y su concepto de "ecología humana" en la sociología estadounidense. Redfield con sus estudios sobre el campesinado y la sociedad folk, y sobre la transición social y cultural de una sociedad tradicional a una urbana, abrió México al campo de la investigación antropológica de investigadores estadounidenses. Véase al respecto González y Romero (1999). humana y el de ecología cultural propuestos para la geografía por H.H. Barrows como "campo único de la geografía" (Barrows, 1923: 3) y por Carl Sauer, respectivamente. Barrows y Sauer se habían interesado por la adaptación histórica de los pueblos aborígenes al marco geográfico, aunque con ciertas diferencias entre ellos que los distinguen, y ofrecieron sendas vías de estudio a la comprensión desde la geografía de la relación hombre-medio.

\section{De la ecología humana a la cultural, y los paisajes culturales}

Harlan H. Barrows (1877-1960), geógrafo estadounidense, es conocido principalmente por el discurso presidencial titulado Geography as Human Ecology leído ante la Association of American Geographers y publicado en 1923 (Barrows, 1923) que significaba la incorporación de la ecología a la geografía, en el estudio del ser humano en el territorio.

Cuando Barrows presentó este concepto ante la asamblea de la Asociación, ya tenía el campo preparado, como recuerda Capel (1987: 53). Desde la sociología norteamericana, Robert E. Park un sociólogo abierto a los temas geográficos, había abierto camino con el propio término "ecología humana" en un intento de "trasladar los conceptos ecológicos de procedencia naturalista en el [al] estudio de las comunidades humanas" (Capel, 1987: 54). Para este sociólogo, pese a la proximidad en el objeto y los intereses que él mismo reconocía entre la ecología humana y la geografía humana trató de fundamentar desde posturas neokantianas la diferencia entre ambos proyectos intelectuales. Consideró que la ecología humana era una ciencia nomotética, mientras que la geografía humana era idiográfica (Entrikin, 1980: 44; Capel, 1987: 58). Sin embargo, la aportación de Barrows parte de otra tradición intelectual, que tuvo, en los Estados Unidos, un claro antecedente en la obra de E.C. Semple, y así lo trata de justificar en su discurso: no hay en él una sola referencia a Park, aunque la forma como está organizada la exposición y los temas abordados dan a entender que se trataba de defender el campo de estudio de la propia disciplina, la geografía. 
En el contexto de una geografía histórica norteamericana dominada por el determinismo ambiental de Ellen C. Semple y por el discurso de Frederick Turner sobre la frontera, las aportaciones de Barrows a esta especialidad vienen de la necesidad de extender "el método científico para entender los aspectos humanos de la geografía en aquellos estudios de las relaciones entre la cultura y el medio ambiente, en su interacción sobre un área determinada" (Barrows, en Koelsch, 1969: $634)^{14}$. La geografía histórica, según este autor, se debía dedicar a "estudiar las relaciones (hombre-medio) desde las primeras etapas de adaptación y luego considerar las sucesivas etapas a lo largo del tiempo" (Barrows, 1923: 11). La geografía histórica era, pues, la ecología humana a través del tiempo ${ }^{15}$.

Por su parte, las aportaciones de Carl O. Sauer (1889-1975) se han englobado dentro de la geografía cultural, la geografía histórica e incluso la ecología cultural (Mitchell, 2000: 29). Conocido es su interés por temas como la difusión tecnológica y cultural, que aprendió del geógrafo Edward Hahn y que compartió con el antropólogo Robert Lowie, por la identificación de las "áreas culturales" de Kroeber, o sobre el origen del hombre americano y las vías de penetración en el continente.

La atención que Sauer dedicó a los pueblos originarios desde la geografía, requería de una necesaria aproximación a la arqueología y a la antropología, si bien la geografía podía aportar algo fundamental como era el estudio de los paisajes, el equivalente geográfico de las áreas y regiones culturales. Dos fragmentos pueden ser representativos de esta posición. Uno corresponde a un artículo

\footnotetext{
14 En aquella época, los aspectos sociales y humanos de las disciplinas científicas no eran considerados como ciencia. Ciencia eran las naturales, la física y química y las exactas. La geografía física entraba dentro de las ciencias naturales.

15 La propuesta de Barrows, en este sentido, ayudaba a entender la relación entre la presión demográfica, las características y los recursos del territorio. Una idea que fue expresada por Ángel Palerm en su artículo "La civilización urbana" (1952) al asociar los tipos de actividad económica (caza-recolección; agricultura de barbecho; agricultura de regadío), con la densidad demográfica y el desarrollo urbano.
}

publicado en 1944 titulado A Geographic Sketch of Early Man in America donde decía que entre los cometidos de la geografía humana estaban "examinar las organizaciones sociales y sus procesos, las formas y significados de sus localizaciones", así como preguntarse "por qué unas culturas surgen sobre un lugar determinado, por qué de su difusión sobre ciertas áreas, y las causas de su decaimiento" (Sauer, 1944: 529). Más tarde, en una obra sobre el origen de la agricultura y su difusión, este autor decía:

"El geógrafo se interesa en el descubrimiento de patrones de vida diferentes y relacionados, tal como se encuentran en las áreas culturales del mundo. Estos patrones tienen interés y significado en tanto en cuanto aprendemos la forma como han Ilegado a ser. El geógrafo, entonces, está propiamente interesado en cartografiar el arte y los artefactos, conocer de donde vienen y cómo se difunden, y cuáles son los medios cultural y ambiental en los que se desenvuelve" (Sauer, 1955: 1. Traducción libre del autor) ${ }^{16}$.

Para tal tipo de estudios, la aproximación a los conceptos y técnicas de investigación de la antropología social que se estaba desarrollando en Estados Unidos iba a ser fundamental.

La noción de paisaje cultural, esbozado por Barrows en su discurso pero cuyo origen se encuentra en la geografía regional francesa, con Jean Brunhes, y en la geografía alemana con los estudios de Otto Schlüter sobre el paisaje cultural (Landschaftkunde), fue rápidamente aceptada por un Sauer todavía apegado a la geografía física e incorporado como elemento central en su The Morphology of Landscape (1926). Si Barrows pretendía convertir los paisajes culturales en el principal tema que debía enfrentar el

\footnotetext{
${ }^{16} \mathrm{El}$ texto original dice así: "He is interested in discovering related and different patterns of living as they are found over the world culture areas. These patterns have interest and meaning as we learn how they came into being. The geographer, therefore, properly is engaged in charting the distribution over the earth of the arts and artifacts of man, to learn whence they came and how they spread, what their contexts are in cultural and physical environments" (Sauer, 1955: 1).
} 
geógrafo regional, Sauer lo transformó en el concepto alrededor del cual había de girar el análisis geográfico. Comprender la evolución de una región pasaba indefectiblemente por sus paisajes. Debía distinguirse entre el paisaje natural y el cultural y los procesos que los configuraba, a cada uno por separado y conjuntamente.

En una geografía, la estadounidense de los años veinte y treinta, dominada por el determinismo ambiental y cada vez más "en retirada", como denunciaba Sauer (1941: 2), el estudio de los paisajes, en particular de los paisajes culturales, representaba la posibilidad de rastrear las huellas del actuar humano sobre el territorio, conocer "la naturaleza de la transformación humana del medio" (Mitchell, 2000: 28). En definitiva, se trataba de entender el proceso de su configuración. La cada vez mayor atención que se dio a los elementos culturales, por parte de los geógrafos culturales, y la cada vez menor a los procesos de cambio de los paisajes, condujo a Sauer a orientarse más hacia la ecología cultural que empezaba a florecer en ese decenio de los cuarenta y principios de los cincuenta con Julian Steward.

En este contexto intelectual, la atención que despertó México entre los investigadores estadounidenses respondía perfectamente a las expectativas de investigación que se requerían para el desarrollo tanto de los estudios antropológicos, como las que representaba Sauer a través del concepto de paisaje cultural. Las visitas del geógrafo estadounidense a este país con el objeto de hacer observaciones y trabajo de campo, junto con la actividad desarrollada por Robert Redfield y Alfred Kroeber desde mediados de los años veinte inauguraron un camino que habría de ser seguido por numerosos investigadores ${ }^{17}$.

A su vez, también en México se vivían otras necesidades desde el punto de vista de la investigación en antropología. Hacia los años cuarenta y cincuenta del pasado siglo, los antropólogos Pedro Armillas (19141984), Ángel Palerm (1917-1980), junto

\footnotetext{
17 Con respecto a los viajes de Sauer a México, puede consultarse la tabla realizada por Mendoza y Busto (2010: 134).
}

Eric Robert Wolf (1923-1999)18 antropólogo austríaco-estadounidense, afrontaron el reto de tratar de entender el origen y desarrollo de las culturas prehispánicas, principalmente las del centro del país. Sus descubrimientos, marcaron un punto de inflexión en el tipo de investigaciones que en estos campos se practicaba en México al irrumpir con enfoques ligados a la ecología humana y cultural en el funcionalismo dominante de la antropología mexicana de entonces (Medina, 1998: 254) ${ }^{19}$.

\section{Nuevas líneas de aproximación al conocimiento del pasado prehispánico}

Confluyeron en la antropología mexicana de los años cuarenta, diversas propuestas teóricas que trataban de explicar y conciliar el desarrollo agrícola y el aumento poblacional, con el desarrollo urbano y de estructuras centralizadas de gobierno (Estados, ciudadesEstado). Las ideas de Childe, Wittfogel y Steward, expuestas en esos años, sirvieron de base para la introducción de la vertiente culturalista en México. Los temas que abordaron Armillas, Palerm y Wolf, y la forma de hacerlo, eran la respuesta que en México se daba desde la arqueología y la antropología social a varios problemas que estaban preocupando intelectualmente desde mediados del siglo XIX a especialistas diversos de las ciencias sociales, y que tenían que ver con la evolución cultural, la difusión de conocimientos y tecnología, y la formación de organizaciones estatales. Sus observaciones y trabajos son de

18 Sobre Pedro Armillas puede leerse la obra preparada por Teresa Rojas $(1991,2$ vol.) que incluye una selección de sus mejores artículos. De Ángel Palerm, hay varias biografías. He utilizado principalmente Martínez (2000), González (2000 y 2007). Sobre Eric Wolf, puede verse el artículo de Romero y Ávila (1999).

${ }^{19}$ La antropología mexicana de esos años, según Medina, se caracterizaba por su carácter fuertemente nacionalista, que reivindicaba la grandiosidad de las raíces indígenas del pasado y trataba de revalorizar al indio presente y a su cultura. Hay que mencionar que una parte importante de la actividad realizada desde el Museo Nacional tuvo que ver con trabajos arqueológicos que permitieran no solo hallar nuevas ruinas, sino, y sobre todo, reconstruir la grandiosidad y belleza de los monumentos en un afán, en gran parte, educativo. 
gran valor para la arqueología y la etnohistoria, y también para la geografía histórica.

En este contexto, hay que conceder un lugar especial a la aportación conceptual del antropólogo de origen alemán Paul Kirchhoff al identificar Mesoamérica como área cultural, en el sentido kroeberiano del término, a partir de sus rasgos étnicos y culturales expuesta en su artículo Mesoamérica: sus límites geográficos, composición étnica y caracteres culturales $(1943)^{20}$. Sin este marco conceptual, geográfico, que fue tomado críticamente por Armillas, Palerm y Wolf, en mi opinión, sus trabajos difícilmente hubieran tenido la resonancia que tuvieron.

La obra del arqueólogo australiano Vere Gordon Childe What Happened in History? (1942), es considerada como un necesario referente por los mencionados investigadores. En ella, su autor vinculaba la revolución urbana con la producción de excedentes. Childe contribuyó, entre otras cosas, a pensar en la necesidad en el trabajo arqueológico de dejar de lado la monumentalidad de los restos históricos y centrarse en la cotidianiedad, esto es, en la vida de las personas que vivían, trabajaban y alimentaban el funcionamiento de los centros urbanos $y$, finalmente, culturales del mundo (Steward, 1966: 729; Jiménez Villalba, 1995: 161). Tal reflexión parecía dirigida especialmente al trabajo que se estaba realizando en México en este campo, cuyos responsables parecían más preocupados en el descubrimiento de las grandes estructuras y en el uso nacionalista de esos descubrimientos que no en los objetos del día a día.

A estas ideas se añadía el concepto marxista que recuperó Karl Wittfogel, desde fines de los años veinte, de "despotismo oriental" que cristalizó en la obra del mismo nombre años más tarde (1957). Su aportación prin-

\footnotetext{
20 Publicado inicialmente en Acta americana (1943, $N^{\circ}$ 1) tuvo una segunda reedición en el Suplemento de la revista Tlatoani, de 1960 de la Escuela Nacional de Antropología e Historia, con el objetivo, expresado por el propio autor, de alimentar su crítica. Paul Kirchhoff, según explicaba Palerm, no se sustrajo tampoco a la influencia de Childe, Wittfogel y Steward, y fue "a su alrededor" que se organizó la corriente intelectual de honda repercusiones en México (Palerm, 2007: 105).
}

cipal fue la recuperación del concepto de "modo de producción asiático" que, aunque calificado por los economistas liberales ingleses como una peculiaridad de las sociedades orientales, Karl Marx ya había identificado y designado como tal, trabajándolo con cierta profundidad para abandonarlo posteriormente sin darle viso de continuidad. Wittfogel especialista en la civilización china retomó esta noción y la llevó hasta sus extremos, tanto en sus conclusiones políticas, referidas al modelo de sociedad implantada en los países autodenominados de "socialismo real" 21 , como en sus aspectos geográficos: fuera de los núcleos de las civilizaciones china, india y egipcia, pudieron existir otras áreas culturales que hubieran desarrollado un tipo de sociedad, de modelo económico y político, cuya base fuera el agua y las técnicas asociadas tanto para solucionar su escasez como para controlar y aprovechar su exceso. Con el término de "despotismo oriental", "despotismo hidráulico" o, más concretamente, "sociedades hidráulicas", trató el autor alemán de explicar las formas de organización estatal en ciertos núcleos de civilización a partir de un modelo de agricultura de riego ${ }^{22}$.

El enfoque de Wittfogel justificaba una evolución multilineal de las culturas humanas, una conclusión a la que el propio Marx oficialmente parecía negarse a llegar y que,

\footnotetext{
21 Por lo que explica Palerm (2007), Marx vislumbró semejanzas entre el modelo de sociedad oriental basado en el modo asiático de producción y una dirección despótica del gobierno, con lo que podría acontecer con una revolución proletaria como la que se llevó a cabo en Rusia, lo cual le hizo recular y abandonar el análisis de las sociedades orientales. Wittfogel Ilevó a sus últimas consecuencias el análisis abandonado por Marx.

22 En efecto, Wittfogel como sinólogo e inicialmente comunista, comenzó a reflexionar en ciertos artículos respecto del régimen de gobierno implantado a partir de una economía agrícola basado en el regadío (Probleme der chinesichen Wirtschaftgesichte, 1927). Pero fue finalmente, ya en los Estados Unidos que publicó la obra Oriental despotism (1957). La influencia de este autor fue fundamental en la antropología social o cultural mexicana y a él dedicó Palerm su atención durante toda su vida. Al respecto puede verse Palerm (2007), una compilación de artículos de este autor publicados en la revista de la Universidad Iberoamericana "Comunidad" entre los años 1969 y 1970 en una edición preparada por Alba González Jácome (2007).
} 
asimismo en la antropología, costó mucho aceptar $^{23}$. Por aquellos años Julian H. Steward un antropólogo evolucionista estadounidense de línea influida por Kroeber (Harris, 2008: 294) publicó un artículo que fue fundamental para el desarrollo de las ideas de estos tres investigadores. Me refiero a "Cultural Causality and Law" (1949), ampliamente citado por Palerm en sus trabajos. En él cuestionaba la imposibilidad, como habían planteado los seguidores de Franz Boas y los defensores del difusionismo, de establecer un principio causal, o regularidades que devinieran en leyes por decirlo de alguna manera, y paralelismos en la evolución de los diversos grupos culturales de México y del mundo (Palerm, 1952; González Jácome, 2000; Rojas Rabiela, 2001) y apostaba por un evolucionismo multilineal, a saber, la existencia de grupos culturales diversos que habían evolucionado indistintamente a partir de la adaptación cultural a unas condiciones ambientales dadas ${ }^{24}$. De ahí se derivó una nueva línea de estudios en la antropología, la ecología cultural, de raigambre saueriana, por la cual el hábitat natural pasaba a ser una variable dependiente en la determinación de las formas de vida de una población o de una sociedad y de la cual los tres investigadores, Armillas, Palerm y Wolf, fueron sus portavoces ${ }^{25}$.

Con este bagaje teórico, estos antropólogos generaron diversas líneas de investigación que les permitieran reunir un número de datos imprescindibles para esclarecer el desarrollo cultural de los pueblos mesoamericanos, relacionarlo con los recursos

23 Así lo explica Palerm (2007: 58 y ss.), pero también se recoge, por ejemplo, en la comunicación presentada por Wittfogel en el Simposio sobre las civilizaciones del regadío celebrado en Washington (EE. UU.) en 1955, titulada La gran objeción al concepto unilineal de desarrollo: la sociedad hidráulica.

${ }^{24}$ Más allá del hábitat natural, la ecología cultural de Steward apostaba por una relación hombre-medio en el que los recursos que ofrece el medio, la disponibilidad de la tecnología en un momento determinado -en cuanto a conocimientos e instrumentos-y la organización del trabajo, se conformaban como un todo que era relativo a un cierto grupo social.

${ }^{25}$ Un hecho fundamental fue la invitación que Steward hizo a Pedro Armillas y Ángel Palerm, entre otros investigadores, a participar en un Simposio sobre las civilizaciones de regadío, en el año 1953 (Rojas, 2001: 16). naturales disponibles, las técnicas de cultivo y la población existente ${ }^{26}$. Para el caso de Ángel Palerm y de Eric Wolf, se añade su interés por el modo asiático de producción y su aplicabilidad al conocimiento del México prehispánico.

Trabajos como los realizados por Pedro Armillas en los años previos, como $A$ sequence of cultural development in Meso-America (Armillas, 1948), sugerían ya las líneas de investigación en arqueología que guardan relación con los aspectos citados anteriormente y con la geografía histórica:

"Formular un esquema del desarrollo cultural para Mesoamérica (...) debería ser útil para hacer comparaciones con otras áreas y debería estimular el interés en muchos e importantes problemas como la distribución espacial y temporal de diferentes tipos de cultivos, su relevancia comparativa en la vida económica, el significado de los cambios en los instrumentos, y sus consecuencias en los patrones sociales y de asentamientos" (Armillas, 1948: 105. Cursiva y traducción libre del autor).

También Palerm propuso en La civilización urbana (1952) un programa de investigación basado en cuatro puntos, que de forma resumida sería: "1) Estudio de datos etnográficos modernos (...) en el que se expusiera y analizara los efectos sociales y demográficos de las técnicas agrícolas actuales; 2) Deter-

${ }^{26}$ De entre los autores que se dedicaron a los estudios demográficos en el período, sobre todo, del contacto con los españoles destacan S. Cook, W. Borah, L. Simpson. Sus trabajos oscilan entre lo puramente demográfico y los ligados a la ecología humana. Así Sherburne F. Cook y Lesley B. Simpson presentaron en 1948 The Population of Central Mexico in the Sixteenth Century ya en 1948. Cook junto con Woodrow Borah tiene, entre otros, The Population of Central Mexico in 1548. An Analysis of the Suma de Visitas de Pueblos (1958), Indian Population of Central Mexico, 1531-1610 (1960), The aboriginal Population of Central Mexico on the Eve of the Spanish Conquest (1963) y Essays in Population History. Mexico and the Caribbean (1971). Más tarde Cook publicó diversos trabajos como The Historical Demography and Ecology of the Teotlalpan (1949), Soil Erosion and Population in Central Mexico que se encuadran dentro de la línea ecológica de los estudios geográficos. 
minación de las zonas y lugares de regadío indígenas antes del siglo XVI y durante él; 3) Análisis de las situación demográfica y urbana en el siglo XVI y con anterioridad a él; 4) Localización de los puntos de expansión militar mexica" (Palerm, 1952: 186).

La actividad de Armillas, Palerm y Wolf cabe situarla en este contexto teórico mencionado y tuvo como finalidad relacionar el desarrollo agrícola basado en la irrigación, con el aumento demográfico, la aparición de centros urbanos y el entronizamiento de una autoridad central fuerte. Entre sus publicaciones destacamos las de los primeros años por su interés para la geografía histórica. Fue una etapa, desde finales de los años cuarenta hasta mediados los sesenta, de elevada productividad y muy rica en aportaciones y discusiones teóricas.

\section{Aportaciones desde la antropología social a la geografía histórica}

Sobre la aportación de estos tres investigadores a la geografía histórica, hay que decir que en ellos pesaba, sobremanera, el concepto de Mesoamérica propuesto por Kirchhoff, entendido como "área cultural". Los tres coincidieron en la idea de Mesoamérica como "mosaico" climático, étnico (Armillas, 1991, Vol. I: 194; Palerm y Wolf, 1972: 150), "mosaico geográfico, cultural y ecológico" (Palerm y Wolf, 1972: 150) o "mosaico cultural y ecológico" (Palerm, 1967, citado en González Jácome, 2007: 24) para el que los límites geográficos propuestos de $10^{\circ}$ y $22^{\circ} \mathrm{N}$ no eran suficientes como para diferenciarla del resto de las Américas, septentrional o meridional, y había que buscar unos criterios que con mayor precisión les permitiera definirlas. Como expresaban Palerm y Wolf, refiriéndose a la frontera norte de Mesoamérica, la divisoria ecológica, cultural y política no siempre coincidía.

De esta manera, el principal cometido que emprendieron fue el de llenar de contenido a la expresión Mesoamérica como área cultural y marco conceptual. El marco teórico fue otorgado desde la ecología cultural. Y así lo hicieron al tratar de asociar Mesoamérica con unos cultivos (área de domesticación de semillas), un tipo de sistema de cultivo, el basado en las tecnologías para el uso del agua, tanto para organizar el exceso como para gestionar la escasez, que se fundamentarían en el regadío (captación y represamiento, conducciones, etcétera), o en los riegos de humedad (chinampas, principalmente, "el pináculo de la perfección técnica", en materia de uso del agua para fines agrícolas según Armillas) ${ }^{27}$. Unas tecnologías que condujeran, primero, a un cambio en la relación hombre/sociedad- naturaleza/medio (superar el estadio de "parasitismo", en el vocabulario de Childe, para entrar en un estadio de "colaboración", "simbiosis") en el que hombre y naturaleza se vieran mutuamente beneficiados. Tales tecnologías conllevarían un tipo de asentamientos -centros urbanos- y a un tipo de organización política -el Estado-que sería la que dominaría en gran parte del ámbito mesoamericano.

\section{Aportación de Pedro Armillas}

Notas sobre sistemas de cultivo en Mesoamérica: cultivo de riego y humedad en la cuenca del río Balsas (1949) es un texto original dentro de la antropología mexicana ${ }^{28}$. Es resultado, en cierta manera del proyecto que había manifestado en 1948, entre otras cosas, de "conocer la distribución espacial y temporal de los diferentes tipos de cultivos" y profundiza en la relación entre estos cultivos, los sistemas agrícolas empleados y las diversas etapas de desarrollo político y económico de las diversas regiones del área de estudio. Como tratará de mostrar, Mesoamérica se identifica con la supuesta extensión de los sistemas de riego en la época prehispánica ${ }^{29}$,

\footnotetext{
27 Para Armillas, como luego para Palerm y Wolf, Mesoamérica entendida como área cultural pasaba, directamente, por la identificación con los sistemas de riego en la época prehispánica.

28 Publicado originalmente con ese título en Anales del Museo Nacional de México (1949, № 3: 85113). Utilizo la versión publicada en el texto $A r-$ millas: vida y obra, en una edición preparada por Teresa Rojas en 1991.

${ }^{29}$ En este texto entiende Mesoamérica tal como lo propuso Kirchhoff, esto es, como área geográfica, si bien, a lo largo de su trayectoria profesional irá proponiendo límites a tal denominación e, incluso, a calificar como "hipótesis" el listado de factores que ayudarían a su definición -tal como pretendió Kirchhoff que se considerara (Kirchhoff, 1960)-. En
} 
pues, en su opinión, "no podemos estudiar la economía mesoamericana (...) en función solo de los cultivos de temporal" (Armillas, 1991, Vol. I: 162).

El texto está dividido en dos partes. En la primera hace referencia a aspectos generales del clima de Mesoamérica y a los sistemas de cultivo en ella desarrollados, con especial énfasis en los sistemas de irrigación; la segunda está dedicada a la cuenca del río Balsas, a su descripción climática e hidrográfica, y los sistemas de cultivo y tipos de cultivo históricos que los documentos históricos han registrado para los núcleos de población de la región del Balsas.

Armillas concede una particular relevancia al factor climático por su directa relación con el desarrollo de sistemas agrícolas que requieren del empleo de tecnologías hidráulicas. Las características del relieve de toda el área mesoamericana más su posición latitudinal en el globo, hacen de ella un "mosaico climático" paralelo al "mosaico étnico" que sobre Mesoamérica se ha asentado. Así, dice:

"En un estudio de la distribución de los sistemas y tipos de cultivos en nuestra área, es necesario tener muy presente la complejidad climática de Mesoamérica, no solo las diferencias regionales (...) sino también las diferencias locales. (...) Para estudiar la interrelación entre ambiente y cultura, el grado de importancia relativa de los factores climático y étnico en la determinación de la historia cultural de Mesoamérica, es necesario tener en cuenta esa complejidad climática no menos que la complejidad étnica" (Armillas, 1991, Vol. I: 194).

el texto posterior que comentaremos a continuación, de 1961, definirá Mesoamérica, como aquella área cultural en la que las culturas maya y mexica, desempeñaron en los últimos tiempos un papel rector: "El término Mesoamérica se utiliza actualmente para referirse a un área cultural que abarcaba las antiguas civilizaciones maya y mexicana (...) y que en el pasado se consideraron como desarrollos diferentes. Su unidad fundamental es indudable en nuestros días y las diferencias en las estructuras socioeconómicas y en el patrón de asentamientos pueden explicarse satisfactoriamente como adaptaciones ecológicas a medios contrastantes" (Armillas, 1991, Vol. II: 115).
De esta manera, a partir de los datos de clasificación climática de Köppen para México, asocia los diversos sistemas de cultivo y las principales plantas empleadas a partir de referencias históricas y del uso de la toponimia como documento histórico.

La investigación llevada a cabo en el artículo citado preludia un trabajo posterior de gran calidad y de mucho interés para la geografía histórica americana -con especial atención a Mesoamérica-, elaborado cuando estaba en la Universidad de Michigan (EE.UU.). Se trata del documento Land-use in pre-Columbian America una contribución a la obra A History of land Use in Arid Regions (Stamp, 1961) presentada dentro del programa marco de la UNESCO de investigación en las zonas áridas del mundo (Arid Zone Research) ${ }^{30}$. Arid Zone Research nació de una resolución que se tomó durante la celebración en Beirut de la Conferencia General de esa organización del año de 1948 con el ánimo de potenciar iniciativas que pusieran en valor productivo una parte importante de las tierras del mun$\mathrm{do}^{31}$. El volumen en el que participó Armillas era "un intento de averiguar qué lecciones podrían obtenerse del pasado, que ayudaran a comprender el presente y preparar un plan de futuro" (citado en Woodbury, 1963: 410) y en él se presentaron estudios de diversas partes del mundo, entre las que figuraba América elaborado por Armillas ${ }^{32}$.

En esta obra repasaba para todo el continente temas todavía conflictivos como las diversas ideas con respecto a la llegada del hombre a América, sobre la domesticación

30 Hemos usado indistintamente la versión original de 1961 publicada en línea (Armillas, 1961), como la versión castellana en formato impreso. La traducción propuesta en la obra editada por Teresa Rojas (1991) tiene algunos pequeños defectos de traducción que he tratado de subsanar consultando la versión original.

31 México formaba parte desde 1950 del Consejo Internacional Interino de investigación sobre zonas áridas, junto con otros siete países como Egipto, Francia, Israel, India, Reino Unido, Estados Unidos.

32 De los trabajos comprendidos en el mencionado volumen, Woodbury en una reseña de la obra aparecida en American Anthropologist (Woodbury, 1963, p. 410-414) elogiaba el texto de Armillas y lo calificaba como el más completo y más actualizado en el marco teórico vigente en aquellos años. 
de las primeras plantas y el lugar o los lugares en donde ese proceso debió iniciarse, así como su interpretación para el ámbito mesoamericano de las ideas de Gordon Childe acerca del origen de los centros urbanos y de organizaciones políticas asimilables a Estados ligados al desarrollo de la agricultura. Para el caso de Mesoamérica, opinaba, fue el establecimiento de la agricultura de regadío lo que permitió afirmar "las bases para un eventual desarrollo de la civilización" sobre todo en los ámbitos semiáridos y áridos de la región (Armillas, 1961: 256).

"Fue solo mediante el aumento de los cultivos y el mejoramiento de las técnicas agrícolas que los indígenas americanos fueron capaces de dominar muchas de las regiones áridas y semiáridas del continente, y en dos casos independientes pudieron construir, basándose en la agricultura intensiva, civilizaciones comparables a las del preclásico del Viejo Mundo: Mesopotamia, Egipto, el Indo y el norte de China" (ArmiIlas, 1961: 256. Traducción libre del autor).

La cultura es entendida como un factor de primera magnitud en el proceso de adaptación del ser humano al medio.

"El hombre ha empleado mucho tiempo en adaptarse culturalmente a medios variados y cambiantes y para poder modificar con sus obras los paisajes americanos" (ArmiIlas, 1961: 256. Traducción libre del autor).

Con ella se superaron "las barreras ecológicas", las limitaciones, que la naturaleza imponía al ser humano. Y el surgimiento de la agricultura, pero en particular, la agricultura establecida, supuso para él un cambio fundamental en la relación del hombre con su medio (Armillas, 1961: 256).

\section{Aportaciones de Palerm y de Wolf}

El artículo de Pedro Armillas de 1948 A sequence of cultural development in MesoAmerica, ya citado, sirvió de acicate y de base a las investigaciones de Palerm sobre La civilización urbana y su dedicación a los regadíos prehispánicos ${ }^{33}$ y en Wolf, a la colaboración

\footnotetext{
33 Las obras de Palerm se insertan en un primer momento dentro de la ecología humana. Posterior-
}

con Palerm y, aparte, sus trabajos sobre la población indígena actual y acerca de la organización campesina, entre otros escritos.

Distribución del regadío en el área central de Mesoamérica ${ }^{34}$ (1954), realizado por Palerm, y "Potencial ecológico y desarrollo cultural en Mesoamérica" (1957), de Palerm y Wolf, resultado de una exposición ante la Sociedad antropológica de Washington y publicada originalmente en una obra titulada Studies in Human Ecology, profundizan la labor realizada previamente por Armillas en sus Notas sobre sistemas de cultivo en Mesoamérica (1949). Si ya en ella, Armillas hizo una recopilación de noticias de regadíos aplicada a la cuenca del río Balsas, Palerm realizó una labor parecida en "Distribución del regadío", y ambos textos representaron un importante impulso a la reivindicación de Mesoamérica como área cultural.

"Distribución del regadío" es un detallado seguimiento de las noticias históricas sobre la presencia, o no, de regadío en el México central. El principal objetivo de este trabajo era el de contestar con argumentos la idea extendida entre antropólogos y geógrafos estadounidenses, como Kroeber y Sauer, sobre la poca relevancia de los sistemas de irrigación en esta región, la inexistencia de núcleos urbanos $y$, por tanto de algo asemejable a "civilización" y, posteriormente, una vez mostrada su existencia, asociarlos con las ideas de Childe y Wittfogel, acerca del origen de núcleos urbanos -ya no más considerados como centros únicamente ceremoniales- $y$, en consecuencia, de una civilización urbana, también en Mesoamérica.

Dividido en cuatro apartados, el trabajo es una larga lista de localidades ordenadas por estados en los que históricamente existen refe-

mente, con el influjo de Julian Steward a través de Wolf, se empieza a hablar de ecología cultural. Sin embargo, antes de esta incorporación de los citados conceptos, en México había autores que ya habían reflexionado acerca de la relación hombre-medio y sobre problemas geográficos.

34 Publicado inicialmente en Ciencias Sociales (Vol. 5, № 25 y 26), fue reeditado años más tarde, en 1972, bajo el título Distribución geográfica de los regadíos prehispánicos en el área central de Mesoamérica, en Palerm y Wolf (1972: 30-64). Las alusiones a este trabajo como a "Potencial ecológico..."serán referidas a esta última edición. 
rencias de riego. Iba acompañado de un mapa a pequeña escala con la ubicación aproximada de todas las poblaciones de la zona central de la República mexicana que presentaban, para el momento del contacto entre europeos y amerindios, sistemas de riego.

Pocos años después, en su conferencia ante la Sociedad Antropológica de Washington, titulada Potencial ecológico y desarrollo cultural en Mesoamérica ${ }^{35}$ (1957), Palerm junto con Wolf hicieron una propuesta de delimitación del área mesoamericana, en sus fronteras septentrional y meridional, así como unas subdivisiones "fisiográficas" de todo el conjunto con sus "tipos ecológi$\cos ^{\prime \prime}$, que les permitieran comprender mejor la relación entre el potencial ecológico con el desarrollo cultural alcanzado en la región. Para ello, dividieron en tres grandes zonas el área mesoamericana (altiplano central, altiplano austral y altiplano del sureste) con sus subdivisiones, y propendieron a describirlas desde el punto de vista del relieve, del clima, los sistemas agrícolas empleados, las densidades de población probables, entre otros. Particular relevancia concedieron al criterio que denominaron "área clave" con el que designaban "aquellas zonas que han desempeñado un papel dominante a lo largo de un período de desarrollo" (Palerm y Wolf, 1972: 164).

Este trabajo puede entenderse como un avance con respecto al anteriormente descrito Distribución del regadío en el área central de Mesoamérica. No trataron únicamente de localizar el riego, sino también de buscar factores explicativos que, entre otras cosas, permitieran definir con mayor rigor el concepto de Mesoamérica, por un lado y, por otro, mostrar la aplicabilidad de las ideas de Childe, Wittfogel y Steward en esta región geográfica.

\section{De la crítica a la continuidad de los estudios sobre el regadío}

Los resultados de las investigaciones y las ideas expuestas por estos tres autores en sus publicaciones no fueron ajenos a las críticas que diversos investigadores formularon a las tesis de Wittfogel y de Steward, principalmen-

35 Utilizo la versión publicada en Palerm y Wolf (1972: 149-205). te. Entre sus ideas, había una que se basaba en la conexión causal entre la complejidad de la construcción de sistemas de regadío de grandes dimensiones y la existencia de una autoridad política centralizada asimilable a una organización estatal, que fuera capaz de organizar ingentes masas humanas y coordinarlas en un esfuerzo común. El "modo asiático de producción" en la interpretación de Wittfogel en su aplicación a Mesoamérica y que Palerm tradujo como modelo teórico que había de contrastarse y verificar, devino referente para orientar las investigaciones ${ }^{36}$. Este modelo fue apoyado por investigadores como William Sanders y Barbara Price en su Mesoamerica: The Evolution of a Civilization (1968) y por otros tantos arqueólogos estadounidenses ${ }^{37}$.

Las críticas estuvieron presentes desde las primeras propuestas a mediados de los años cincuenta. ¿Qué fue antes, la construcción del regadío o la organización política centralizada? ¿Qué fue causa y qué consecuencia? ¿Fue necesaria la presión sobre los recursos de una población creciente, urbana, desvinculada de la agricultura -una clase burocrática- para que se desarrollaran las técnicas de manejo del agua? (Mitchell, 1973: 532).

Molestaba, entre otras cosas, la conexión "mecánica", un determinismo ambiental arreglado, entre un ambiente árido, la autoridad necesaria y la construcción de regadíos. Como manifestaba Murphy "Quizás nuestro problema real es el modelo mecanicístico de causalidad que lleva a buscar las causas en un momento distinto anterior al efecto" (Murphy, en Mitchell, 1973: 533. Traducción libre del autor) y opinaba que, muy posiblemente, ambas cosas habían ido de la mano.

\footnotetext{
36 Wittfogel propuso cuatro condiciones básicas para la implantación de este modelo: 1) Un grado de desarrollo de las fuerzas productivas por encima del nivel de subsistencia; 2) Un medio, no solo donde nazca la agricultura, sino que debe de estar regido por la escasez o el exceso de agua que obligue al desarrollo de técnicas de manejo hidráulicas; 3 ) Dimensiones considerables, geográfica y técnicamente, de las obras de irrigación; 4) Un modelo sociopolítico de carácter estatal en el que el estado deviene promotor, regulador y conservador de los sistemas hidráulicos (Palerm, 2007: 87-89).

37 Entre los trabajos recientes puede verse Doolittle, 1990.
} 
William Mitchell apostaba, en el artículo citado en el que revisaba la hipótesis de Wittfogel y Steward, por una reformulación de la hipótesis hidráulica de la siguiente manera: "Si se da una dirección centralizada de las actividades de irrigación en medios áridos o semiáridos habrá en correspondencia un aumento del poder político centralizado en otras áreas de la vida social" (Mitchell, 1973: 534) y abogaba para que, sin menoscabo de la relevancia de la irrigación, se pudiesen hallar otros factores que pudieran explicar el origen de los Estados en Mesoamérica y otros ámbitos geográficos. Es esta última sugerencia la que, en mi opinión, puede tener aplicación para comprender esa etapa de la historia mexicana contemporánea en la que se apostó por la irrigación a gran escala con el apoyo directo del Estado.

La aportación de Armillas, Palerm y Wolf, como puede comprenderse, no se quedó en el México prehispánico. La labor de investigación emprendida por ellos no quedó interrumpida sino, más bien, inauguró una veta que ha tenido continuidad en la antropología social de México hasta nuestros días ${ }^{38}$. Sus conclusiones son de gran valor para la arqueología y la etnohistoria, y para la geografía histórica: se han tratado de reconstruir las características del entorno y el paisaje en diversas partes de la geografía del México prehispánico, la red urbana y la de regadíos y con ella el uso humano del territorio, y comprender la geografía política e histórica de las culturas mesoamericanas. También brinda otras posibilidades de comprensión al proceso de conformación de los estados nacionales actuales y otra forma de pensar el territorio

\footnotetext{
${ }^{38}$ En ella se situarían los participantes del Seminario de Etnohistoria del valle de México (1973-1976) del que surgieron numerosos estudios sobre "la utilización prehispánica y colonial del riego" (Boehm, 1986: 21). Los trabajos contenidos en Nuevas noticias sobre las obras hidráulicas prehispánicas y coloniales en el Valle de México (Rojas, Strauss, Lameiras, 1974), el libro Historia de la agricultura, de Rojas y Sanders (1985), el Coloquio sobre agricultura indígena: pasado y presente (1986) coordinado por Teresa Rojas y Efraín Hernández, del que surgió un libro con ese mismo título, o el de Briggitte Boehm, Formación del Estado en el México prehispánico (1986), son solo los exponentes de una obra que no ha dejado de dar frutos y cuyo interés para el geógrafo-histórico está fuera de toda duda.
}

a partir de la ecología cultural: formular una hipótesis de trabajo que permita comprender, en última instancia, la correspondencia equilibrada que debería de haber entre los principales términos de su problema, la relación del hombre con el medio y el papel de la cultura -como quiera que sea definida- en ella. Como lo ha planteado Fábregas, "Cada sociedad utiliza una estrategia de adaptación para manejar su medio ambiente, explotarlo a su favor y garantizar la continuidad de la sociedad misma" (Fábregas Puig, 2002: 8). Descubrir quién o qué está detrás de esta estrategia y los beneficios, materiales o espirituales, que se puedan obtener es uno de los problemas que deben ser resueltos.

La mención de los grandes proyectos hidráulicos llevados a cabo desde finales del siglo XIX nos pueden servir como colofón, como aplicación de las tesis ya explicadas de los antropólogos y plantear, una vez más, la necesidad de una geografía histórica que "dé respuesta a los problemas del mundo actual" que destacaba Horacio Capel hace ya un tiempo (Capel, 2006). En este sentido, las cuatro condiciones expuestas por Wittfogel de las sociedades hidráulicas, a saber, Irrigación, Aumento demográfico, Aparición -consolidación, más bien en los años finales del siglo XIX-de los centros urbanos existentes, y Autoridad central fuerte, pueden fácilmente entreverse en la historia del México contemporáneo. Así, de forma abreviada, y en relación con la conformación del Estado moderno, en el México postrevolucionario, las grandes obras de irrigación tuvieron un momento de auge, si bien ya en tiempos finales de la presidencia del general Porfirio Díaz ya se estaban reclamando y estaban en la boca de políticos y agraristas del momento (Aboites, 1998 y 2000; Sunyer, 2006). Fue una época en la que el Estado asumió un papel protagónico en pro del "interés nacional", recuerda Aboites (1994), en un contexto de fortalecimiento político de la presidencia de la República y del aparato estatal; que animó a ingenieros civiles y agrónomos, así como a otras corporaciones y grupos de científicos, a seguir el llamado de la construcción de un nuevo proyecto de Nación, que condujera a la ocupación agrícola del árido del país.

Esta implicación de los ingenieros hidráulicos ha sido entendida como "corolario de 
una decisión política de largo alcance, referido al control estatal de la innovación tecnológica en materia hidráulica" (Aboites, 2000: 96), en la que se substituía, en aquellos tiempos, al propietario como potencial innovador por el técnico al servicio del Estado en esa labor. En términos de la ecología cultural, se trataba, según Fábregas, de "una estrategia de adaptación [del Estado] para manejar su medio ambiente, explotarlo a su favor y garantizar la continuidad de la sociedad misma". La existencia de un poder fuerte y centralizado, como fueron el de Porfirio Díaz o el surgido tras la Revolución, actuaría como "factor estratégico para determinar la adaptabilidad" y determinar "las estrategias adaptantes" (Fábregas Puig, 2002: 8 y 11). Así, la pertinencia de que fuera el gobierno federal el que asumiera, al menos, una parte importante de la inversión, se convertía en la opción viable para la construcción de un Estado fuerte basado, durante el porfiriato -en el ideario liberal-y durante la época revolucionaria y posteriormente, un proyecto de Estado marcadamente social, y menos socialista.

No fue México, para estos años a los que hacemos alusión, un caso excepcional. Hubo en el contexto internacional muchos países en los que el Estado adquirió tintes autoritarios o dictatoriales y en los que emprendió grandes iniciativas de construcción de sus respectivos territorios nacionales: carreteras y vías de comunicación, telecomunicaciones, regadíos, puertos... necesarias para su integración territorial y para legitimarse ante la población como benefactores, a cambio, eso sí, de una cierta pérdida en las libertades individuales, como ha propuesto Harris (1999). En este sentido, y desde la perspectiva de la ecología cultural, la construcción histórica del territorio nacional y, con él, la del Estado moderno, puede interpretarse como "la existencia y operación de una ecología cultural políticamente decidida y dirigida" (Fábregas Puig, 2009: 171-172)39. Unas políticas que, en entornos poco avenidos con la democracia política que muchos de los países tienen, asignan las funciones que cada parte

\footnotetext{
39 Este comentario lo hizo a propósito de la obra de Boehm (1986) en relación con lo que consideraba que era su aportación en el estudio del proceso de formación del estado en tiempos prehispánicos.
}

del territorio ha de cumplir en relación con la supervivencia del propio Estado.

\section{Referencias bibliográficas}

ABOITES, L. Apuntes para una historia de los usos del agua en México en los siglos XIX y XX. Estudios Sociales, 1994, Vol. 15, № 9, p. 221-235.

ABOITES, L. El agua de la nación: una historia política de México (1888-1946). México: Centro de Investigaciones y Estudios Superiores en Antropología Social (CIESAS), 1998.

ABOITES, L. Optimismo nacional: geografía, ingeniería hidráulica y política en México (1926- 1976). En: MENTZ, B. (coordinador). Identidades, estado nacional y globalidad. México, siglos XIX y XX. México: Centro de Investigaciones y Estudios superiores en Antropología Social (CIESAS), 2000, p. 95- 152.

ARMILLAS, P. A sequence of cultural development in Meso-America. Memoirs of the Society for American Antropology, 1948, № 4, p. 15-111.

ARMILLAS, P. Notas sobre sistemas de cultivo en Mesoamérica: cultivo de riego y humedad en la Cuenca del río Balsas. En: ROJAS, T. (editor). Pedro Armillas: vida y obra. México: Centro de Investigaciones y Estudios Superiores en Antropología Social, 1991, Vol. 1, p. 159- 192.

ARMILLAS, P. Land Use in pre-Columbian America. In: DUDLEY STAMP L. A History of Land Use in Arid Regions. Arid Zone Research, Paris: UNESCO, 1961, Vol. 17, p. 255-276. Disponible en internet: http:// es.scribd.com/doc/66240362/Arm-III-as-1961

BARROWS, H. Geography as human ecology. Annals. Association of American Geographers, 1923, Vol. XIII, p. 1-14.

BERDOULAY, V. Perspectivas actuales del posibilismo. De Vidal de la Blache a la ciencia contemporánea. Geocrítica. Cuadernos críticos de Geografía humana, 1983, Año VIII, $N^{\circ} 47$. Disponible en internet: http://www.ub.edu/geocrit/geo47.htm 
BOEHM DE LAMEIRAS, B. La formación del Estado en el México prehispánico. Zamora: El Colegio de Michoacán, 1997.

BOEHM SCHOENDUBE, B.; DURÁN JUÁREZ, J.M.; SÁNCHEZ RODRÍGUEZ, M. y TORRES RODRÍGUEZ, A. (coordinadores). Los estudios del agua en la cuenca LermaChapala-Santiago. Zamora: El Colegio de Michoacán/ Centro Universitario de Ciencias Sociales y Humanidades de la Universidad de Guadalajara, 2002.

CAPEL, H. La geografía histórica y la respuesta a los problemas del mundo actual. Clausura del VIII Coloquio Internacional de Geocrítica. Scripta Nova. Revista electrónica de geografía y ciencias sociales, 2006, Vol. X, No 218 (96). Disponible en internet: http://www.ub.es/geocrit/sn/sn-218-96.htm

DOOlITTLE, W. Canal Irrigation in Prehistoric Mexico. The Sequence of Technological Change. Austin: University of Texas Press, 1990.

FÁBREGAS PUIG, A. La formación histórica de una región. Los Altos de Jalisco. México: Centro de Investigaciones y Estudios Superiores en Antropología Social, 1986.

FÁBREGAS PUIG, A. La ecología cultural política y el estudio de regiones en México. Revista de dialectología y tradiciones populares, 2000, Vol. LXIV, N 1, p. 167- 176.

FÁBREGAS PUIG, A. y TOMÉ MARTÍN, P. Regiones y fronteras. Una perspectiva antropológica. Zapopan: El Colegio de Jalisco/ Secretaría de la Educación Pública, 2002.

GARCÍA MARTÍNEZ, B. En busca de la geografía histórica. Relaciones. El Colegio de Michoacán, 1998, Vol. XIX, N 75, p. 25-58.

GARCÍA MARTÍNEZ, B. El desarrollo regional y la organización del espacio, siglos XVI al XX. In: SEMO, E. (coordinador). Historia económica de México. México: Universidad Nacional Autónoma de México/ Océano, 2004.
GARCÍA MARTÍNEZ, B. Las regiones de México. Breviario geográfico-histórico. México: El Colegio de México, 2008.

GONZÁlEZ JÁCOME, A. Notas sobre las concepciones de Ángel Palerm acerca del ambiente y la agricultura. Ciencia Ergo Sum, 2000, Vol. 7, № 2, s/p. Disponible en internet: http://redalyc.uaemex.mx/ pdf/104/10401817.pdf

GONZÁLEZ JÁCOME, A. Estudio introductorio a Ángel Palerm. Agua y agricultura. La discusión con Karl Wittfogel sobre el Modo Asiático de Producción y la construcción de un modelo para el estudio de Mesoamérica. México: Universidad Iberoamericana, 2007, p. 15-47.

GUZMÁN BETANCOURT, I. Prólogo. In: SAUER, C. Aztatlán. México: Siglo XXI, 1998, p. IX-XXII.

HARRIS, M. El desarrollo de la teoría antropológica. Una historia de las teorías de la cultura. México: Siglo XXI, 2008.

HARRIS BUCHER, G. Releyendo a Karl Wittfogel y su despotismo oriental. Estudio comparativo del poder autoritario. Revista de Estudios histórico-jurídicos, 1999. Disponible en internet: http://www.scielo.cl/ scielo.php?script=sci_arttext \&pid=S0716$54551999002100017 \& \operatorname{lng}=\mathrm{es} \& n r m=i s o$

HEFFERNAN, M. The future of Historical Geography. Journal of Historical Geography, 1997, Vol. 23, No 1, p. 1-2.

HIERNAUX, D. (director). Construyendo la Geografía humana. México: Anthropos editorial, 2010.

JIMÉNEZ VILLABA, F. La teoría de las revoluciones en Vere Gordon Childe. Anales del Museo de América, 1995, № 3, p. 161164.

KIRCHHOFF, P. Mesoamérica: sus límites geográficos, composición étnica y caracteres culturales. Tlatoani, 1960, $\mathrm{N}^{\circ}$ 3. Disponible en internet: http://.alfinliebre.blogspot.com

KOELSCH, W. The Historical Geography of Harlan H. Barrows. Annals of the Associa- 
tion of American Geographers, 1969, Vol. 59, $\mathrm{N}^{\circ} 4$, p. 632-651.

LUIS, A. y URTEAGA, L. Estudio del medio y Heimatkunde en la geografía escolar. Geocrítica. Cuadernos críticos de Geografía humana, 1982, Año VII, Nº 38. Disponible en internet: http://www.ub.edu/geocrit/geo38.htm

MARTÍNEZ SALDAÑA, T. Modelos de desarrollo local. Una visión utópica de Ángel Palerm Vich. Ciencia Ergo Sum, 2000, Vol. 7, $N^{\circ} 3$, p. 229- 234.

MEDINA, A. Origen y formación del Estado en Mesoamérica. México: Universidad Nacional Autónoma de México-Instituto de Investigaciones Antropológicas, 1986.

MEDINA, A. Recuentos y figuraciones: ensayos de antropología mexicana. México: Universidad Nacional Autónoma de México-Instituto de Investigaciones Antropológicas, 1998.

MENDOZA, H. y BUSTO, K. La geografía histórica de México, 1950- 2000. En: HIERNAUX, D. (director). Construyendo la Geografía humana. Rubí: Anthropos editorial, 2010, p. 132- 151.

MITCHELL, D. Cultural Geography. A Critical Introduction. Malden: Blackwell Publishers Inc., 2000.

MITCHELL, W. The Hydraulic Hypothesis. A Reappraisal. Current Anthropology, 1973, Vol. 14, N ${ }^{\circ}$ 5, p. 532-534.

MONCADA MAYA, J. La geografía histórica en el Instituto de Geografía de la Universidad Nacional Autónoma de México. Biblio $3 W$, Revista bibliográfica de geografía y ciencias sociales, 2004. Disponible en internet: http://www.ub.es/geocrit/b3w-500.htm

PALERM, A. La civilización urbana. Historia mexicana, 1952, Vol. 2, №2, p. 184-209.

PALERM, A. Distribución geográfica de los regadíos prehispánicos en el área central de Mesoamérica. En: PALERM, A y WOLF, E. Agricultura y civilización en Mesoamérica. México: Secretaría de Educación Pública, SEPSetentas, 1972, p. 30- 64.
PALERM, A. Agua y agricultura. La discusión con Karl Wittfogel sobre el Modo Asiático de Producción y la construcción de un modelo para el estudio de Mesoamérica. México: Universidad Iberoamericana, Edición preparada y estudio introductorio Alba González Jácome, 2007.

PALERM, A y WOLF, E. Potencial ecológico y desarrollo cultural en Mesoamérica. En: PALERM, A y WOLF, E. Agricultura y civilización en Mesoamérica. México: Secretaría de Educación Pública, SEPSetentas, 1972, p. 149-205.

RITTER, C. Géographie générale compare, ou Étude de la terre dans ses rapports avec la nature et avec l'histoire de l'homme pour servir de base à l'étude et à l'enseignament des Sciences physiques et historiques. Bruxelles: Société Typographique Belge Adolph Wallen et Compagnie, Traduit par Edouard Desor, Eugène Buret (i.e. Antoine Eugène), 1838. Disponible en internet: http://books.google.com.mx/bkshp?hl=es

ROBINSON, D. Historical Geography in Latin America. In: BAKER, A.R.H. Progress in Historical Geography. Nueva York: David and Charles Publishers, 1972, p. 168-186.

ROJAS RABIELA, T. Pedro Armillas: vida y obra. México: Centro de Investigaciones y Estudios Superiores en Antropología Social (CIESAS), 1991, 2 Vol.

ROJAS RABIELA, T. La tecnología agrícola. In: MANZANILLA, L. y LÓPEZ LUJÁN, L. Historia antigua de México. Vol. IV Aspectos fundamentales de la tradición cultural mesoamericana. México: Consejo Nacional para la Cultura y las Artes (CONACULTA)/ Instituto Nacional de Antropología e Historia/ Universidad Nacional Autónoma de México, 2001, p. $13-68$.

ROJAS RABIELA, T. y SANDERS, W. (editores). Historia de la agricultura. Época prehispánica, siglo XVI. México: Instituto Nacional de Antropología e Historia, 1985.

ROJAS RABIELA, T.; STRAUSS, R. y LAMEIRAS, J. Nuevas noticias sobre las obras hidráulicas prehispánicas y coloniales en el Valle de México. México: Secretaría de Edu- 
cación Pública/Centro de Investigaciones Superiores-Instituto Nacional de Antropología e Historia, 1974.

ROMERO CONTRERAS, T. y ÁVILA RAMOS, L. Eric Wolf: humanista y científico social del siglo XX. Ciencia Ergo Sum, 1999, Vol. 6, No 3, p. 322-328.

RUCINQUE H. F. y VELÁSQUEZ, A.L. Geografía e historia: ¿reactivación de antiguas relaciones interdisciplinarias? Revista de Relaciones Internacionales. Estrategia y seguridad, 2007, Vol. 2, №2, p. 127-148.

SAUER, C. Foreword to Historical Geography. Annals of the Association of American Geographers, 1941, Vol. XXXI, Nº 1, p. 1- 24.

SAUER, C. Agricultural Origins and Dispersals. Nueva York, The American Geographical Society, 1952. Disponible en internet: http://www.archive.org/stream/agriculturalorig033518mbp/agriculturalorig033518mbp_ djvu.txt

SAUER, C. A Geographic Sketch of Early Man in America. Geographical Review, 1955, Vol. 34, No 4, p. 529-573.

SAUER, C. Aztatlán. México: Siglo XXI, 1998.

STAMP, L.D. A History of Land Use in Arid Regions. Arid Zone Research, 1961, Vol. 17.

STEWARD, J. Cultural Causality and Law. A Trial Formulation of the Development of
Early Civilizations. American Anthropologist, 1949, Vol. 51, № 1, p. $1-27$.

STEWARD, J. Toward Understanding Cultural Evolution. The evolution of Urban Societies: Early Mesopotamia and Pre-Hispanic Mexico by Robert McC. Adams. Science, 1966, Vol. 153, No3737, p. 729-730.

SUNYER MARTíN, P. Temporal y regadío en el agro mexicano. Política y agricultura en el México de principios del siglo XX. Scripta Nova. Revista electrónica de geografía y ciencias sociales, 2006, Vol. $\mathrm{X}, \mathrm{N}^{\circ} 218$ (05). Disponible en internet: http://www.ub.es/geocrit/sn/sn-218-05.htm

SUNYER MARTÍN, P. La geografía histórica y las nuevas tendencias en la geografía humana. En: LINDÓN, A. y HIERNAUX, D. (directores). Los giros de la Geografía humana. Desafíos y horizontes. Rubí: Anthropos editorial, 2010, p. 143- 173.

SUNYER MARTíN, P. Tendencias de la Geografía histórica en México. Biblio $3 W$. Revista Bibliográfica de Geografía y Ciencias Sociales, 2011, Vol. XVI, No 922. Disponible en internet: http://www.ub.es/geocrit/b3w922.htm.

TRINDELL, R. Franz Boas and American Geography. The Professional Geographer, 1969, Vol. XXI, N 5, p. 328-331.

WOODBURY, R. Reseña a "A History of Land Use in Arid Regions". American Anthropologist, 1963, Vol. 65, N², p. 410-414. 